\title{
Disseminated Mycobacterium avium complex infection mimicking malignancy in a patient with anti-IFN- $\gamma$ autoantibodies: a case report
}

Yun-Kai Yeh ${ }^{1,2}$, Jing-Ya Ding ${ }^{3}$, Cheng-Lung Ku ${ }^{3,4}$ and Wei-Chih Chen ${ }^{2,5^{*}}$

\begin{abstract}
Background: Disseminated nontuberculous mycobacteria (NTM) infections occur mostly in immunocompromised patients. Therefore, it is difficult to diagnose disseminated NTM infections in patients without history of immunocompromised diseases or using immunosuppressant. Patients with anti-interferon- $\gamma$ (IFN- $\gamma$ ) autoantibodies are vulnerable to intracellular infections, such as disseminated NTM. Currently, there is no widely used and efficient technique for the detection of anti-IFN- $\gamma$ autoantibodies. Herein, we report a case of an apparently healthy patient with disseminated Mycobacterium avium complex (MAC) infection who tested positive for anti-IFN- $\gamma$ autoantibodies.

Case presentation: A 64-year-old non-immunocompromised and apparently healthy Asian male presented to the emergency department with complaints of progressive chest pain for about 6 months and weight loss. A bulging tumour was found in the anterior chest wall. Chest computed tomography showed a lung mass over the right lower lobe and an osteolytic lesion with a soft tissue component at the sternum. Sonography-guided biopsies for the osteolytic lesion and sputum culture confirmed the presence of disseminated MAC infection. In addition, positive test result of anti-IFN- $\gamma$ autoantibodies was noted. The patient was prescribed antibiotics. The lesions over the right lower lobe and sternum attenuated following the antibiotic treatment.

Conclusion: Detection of anti-IFN- $\gamma$ autoantibodies is important among previously healthy people with disseminated NTM infection. Presence of anti-IFN- $\gamma$ autoantibodies may suggest a high risk of severe intracellular infection, such as disseminated NTM infection.
\end{abstract}

Keywords: Nontuberculous mycobacteria, Anti-interferon- $\gamma$ autoantibodies, Sonography-guided biopsy, Mycobacterium avium complex

\section{Background}

Nontuberculous mycobacteria (NTM) are a group of microorganisms ubiquitous in the environment. There are more than 160 species of NTM, of which at least 50 have been associated with pulmonary infectious disease. Unlike Mycobacterium tuberculosis, human-to-human transmission of NTM is not common. It is more difficult

\footnotetext{
* Correspondence: wiji.chen@gmail.com

${ }^{2}$ Department of Chest Medicine, Taipei Veterans General Hospital, Taipei, Taiwan

${ }^{5}$ Institute of Emergency and Critical Care medicine and Faculty of Medicine, Medicine, School of Medicine, National Yang-Ming University, No. 201, Sec. 2, Shih-Pai Rd., Beitou District, Taipei 11217, Taiwan

Full list of author information is available at the end of the article
}

to treat NTM than M. tuberculosis due to drug resistance, and the treatment period usually lasts more than 12 months [1]. Although several studies have revealed NTM infections in both immunocompetent and immunocompromised patients, disseminated NTM infection is usually among immunocompromised patients such as people using long-term immunosuppressants or patients with human immunodeficiency virus (HIV) infection, particularly in those with CD4 counts below 50 cells $/ \mu \mathrm{L}$ [2]. Interferon- $\gamma$ (IFN- $\gamma$ ), which is secreted by natural killer (NK) cells and $\mathrm{T}$ cells, plays a critical role in cellular immunity. Previous studies have suggested that IFN- $\gamma$ autoantibodies may play an important

(c) The Author(s). 2019 Open Access This article is distributed under the terms of the Creative Commons Attribution 4.0 International License (http://creativecommons.org/licenses/by/4.0/), which permits unrestricted use, distribution, and 
role in refractory and recurrent disseminated NTM infections [3]. Therefore, we present a case of a previously healthy patient with disseminated MAC infection who tested positive for anti-IFN- $\gamma$ autoantibodies.

\section{Case presentation}

A 64-year-old Asian male patient presented to the emergency department with complaints of progressive chest pain for about 6 months and weight loss. A review of the patient's medical records revealed a prior history of benign prostatic hyperplasia and hypertension. There was no apparent history of alcohol consumption, smoking, illicit drugs herbs, or immunosuppressants.

A bulging mass was found in his anterior chest wall (Fig. 1). Notable laboratory findings included a white blood cell count of $11,400 / \mu \mathrm{L}$ and a C-reactive protein level of $8 \mathrm{mg} / \mathrm{L}$. Chest computed tomography revealed an osteolytic lesion with a soft tissue component at the sternum mediastinal lymphadenopathy (Fig. 2, arrow), and a lung mass in the right lower lobe (RLL) (Fig. 3, arrowhead). Considering the possibility of lung cancer with mediastinal lymphadenopathy and bone metastasis, a sonography-guided biopsy was performed for the osteolytic lesion over the sternum. The pathological report indicated a focal granulomatous inflammation and necrosis without malignant cells. However, ZiehlNeelsen staining was positive. Tissue culture and two sets of sputum all tested positive for Mycobacterium avium complex (MAC). Thus, MAC infection was suspected. Positive result of anti-IFN- $\gamma$ autoantibodies was noted compared with control samples (Fig. 4). Although the patient did not report any prior history of immunosuppressant use and there was no serological evidence of HIV infection or autoimmune diseases, he was considered to be at risk of disseminated NTM infection due to a positive test result for anti-IFN- $\gamma$ autoantibodies.

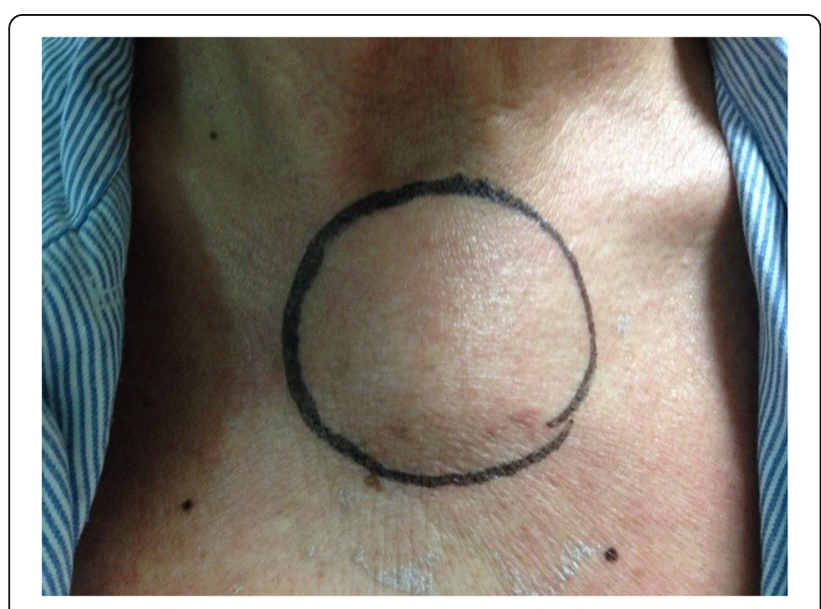

Fig. 1 A bulging mass was found in the anterior chest wall

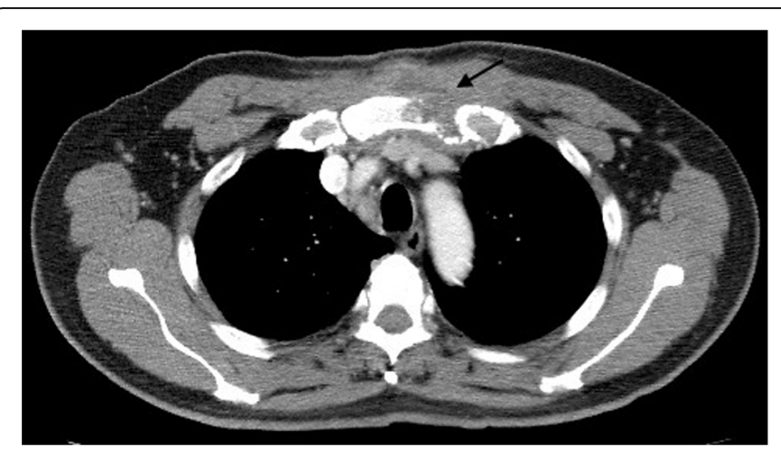

Fig. 2 Arrow: Osteolytic lesion with a soft tissue component at the sternum

Hence, the patient was prescribed with oral form of clarithromycin $500 \mathrm{mg}$ twice daily, oral form of rifabutin $300 \mathrm{mg}$ daily, oral form of ethambutol $15 \mathrm{mg} / \mathrm{kg}$ daily, and intravenous amikacin $15 \mathrm{mg} / \mathrm{kg}$ three times a week for 3 months followed by oral form of clarithromycin $500 \mathrm{mg}$ twice daily, oral form of rifabutin $300 \mathrm{mg}$ daily, and oral form of ethambutol $300 \mathrm{mg}$ daily for another 9 months. The lesions over the RLL and sternum diminished gradually following the treatment (Figs. 5 and 6).

\section{Discussion and conclusions}

\section{Disseminated NTM}

NTM are a group of microbes that are highly prevalent in the environment. Infections resulting from NTM have received more attention recently, especially among patients with chronic renal disease, malignancy, autoimmune disease, and acquired immune deficiency syndrome (AIDS). Nonetheless, the diagnosis of NTM infection can be challenging and biopsy is sometimes necessary to confirm the diagnosis of NTM infection [5]. Lymph nodes are the primary organs that are involved in an NTM infection, followed by osteoarticular system, bone, lung, and skin [4, 6]. Unlike M. tuberculosis (TB), NTM infection does not show human-to-human transmission. Moreover, NTM

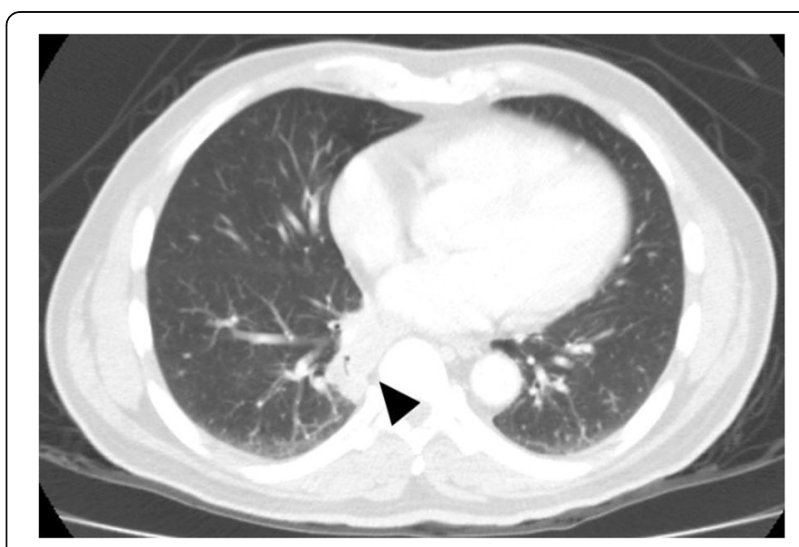

Fig. 3 Arrowhead: Lung mass in the right lower lobe 


\section{IFN- $\gamma$ ELISA (2ug/ml)}

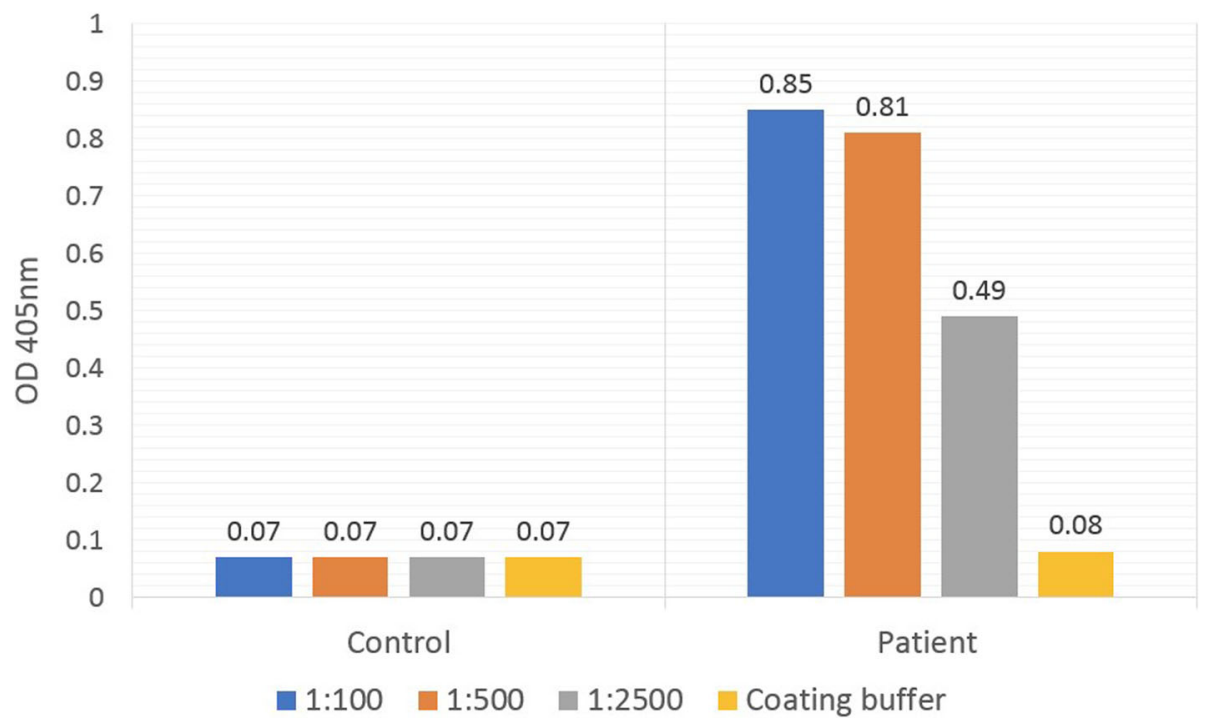

Fig. 4 The presence of anti-IFN- $\gamma$ autoantibodies measured by indirect ELISA. IFN- $\gamma(2 \mu \mathrm{g} / \mathrm{ml}$, BD Bioscience) was coated in each wells; and serially diluted plasma samples from the patient and control samples (dilutions: 1:100, 1:500, and 1:2500) were added into wells. After washing, anti-IFN- $\gamma$ autoantibodies were detected by anti-human lgG antibodies (The Jackson Laboratory) previous described [4]

infection is more difficult to treat compared with infections due to TB because of the high level of resistance to available antimicrobial agents. For example, nearly all NTM species show resistance to pyrazinamide, which is one of mainstay anti-TB drugs. Furthermore, in the case of severe or disseminated NTM, the BTS guidelines recommend intravenous antibiotics, such as amikacin, for at least 3 months. Intravenous antibiotics using may lead to prolong hospitalization stay and influence treatment adherence [7]. Overall, the course of treatment for NTM infections usually lasts for more than 12 months. However, the severity and natural course of infections depend on the immunological status of the host. Long-term antiNTM treatment maybe necessary among patient with incurable immunocompromised status. Disseminated

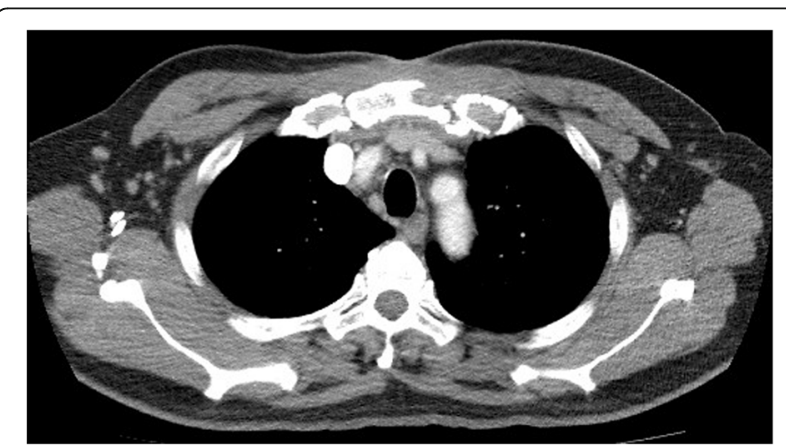

Fig. 5 Osteolytic lesion at sternum diminished gradually after the treatment
MAC infections were very common in patients with advanced AIDS in developing countries before the widespread use of potent antiretroviral therapies [8]. Because disseminated NTM is common and life-threatening in people with advanced AIDS, mycobacterial antibiotic prophylaxis is recommended in patients with HIV infection and whose CD4 count is less than 50 cells $/ \mathrm{mm}^{3}$ [9]. NTM infections occur mostly in immunocompromised patients. Hence, it is difficult to diagnosis disseminated NTM infection in non-immunocompromised patients. In our reported case, a patient without evidence of immunocompromised status was initially considered to have lung cancer instead of disseminated NTM infection.

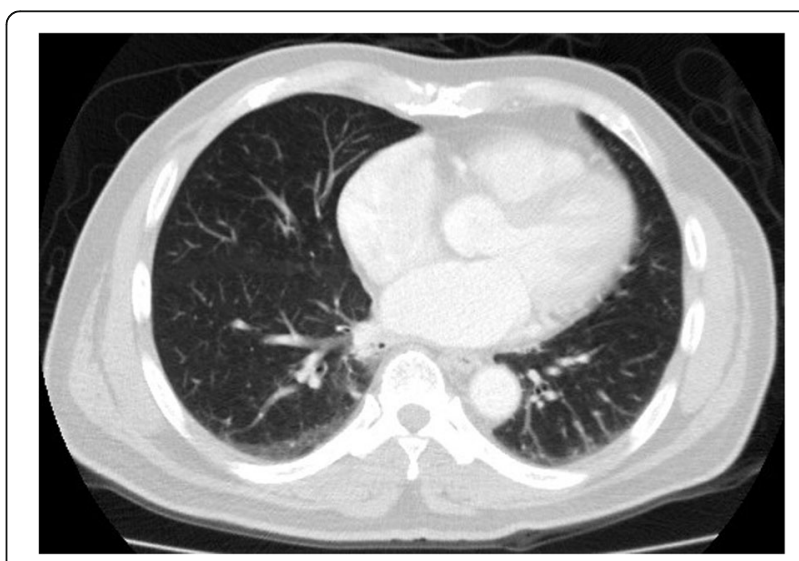

Fig. 6 The size of osteolytic lesion decreased after the treatment 


\section{Anti-IFN- $\gamma$ autoantibodies}

Macrophages generate IL-12/23 that is stimulated by microbial components, such as lipopolysaccharides, and responses to intracellular infection. IFN- $\gamma$ is synthesised by $\mathrm{T}$ cells and NK cells under IL-12/23 stimulation. In turn, IFN- $\gamma$ can activate macrophages, which destroy intracellular microbes. IFN- $\gamma / \mathrm{IL}-12 / 23$ axis plays a crucial role against intracellular infections, such as disseminated NTM infections, salmonellosis, other intramacrophagic bacterial infections, and reactivating latent varicella-zoster virus infection $[3,10]$. Several studies have revealed the impact of IFN- $\gamma / \mathrm{IL}-12 / 23$ axis defects. Kamijo et al. reported a study in which IFN- $\gamma$ receptordeficient mice were vaccinated with Bacillus CalmetteGuerin. Tumoral necrosis factor- $\alpha$ (TNF- $\alpha$ ), IL- $1 \alpha$, and IL- 6 could not be produced after infection in the IFNreceptor-deficient mice. Subsequently, these mice could neither form granulation nor kill mycobacteria because of cell-media immunity defects [11]. Mendelian susceptibility to mycobacterial diseases (MSMD) is a term used to describe a group of patients who are susceptible to mycobacterial diseases because of primary immunodeficiency diseases, including a defect of the interferon- $\gamma$ receptor [12]. Anti-IFN- $\gamma$ autoantibodies have received increasing attention recently and have been associated with disseminated, recurrence, and refractory NTM infections. Patients with high titer of anti-IFN- $\gamma$ autoantibodies are vulnerable to intracellular microbes in a way similar to MSMD. High titer of anti-IFN- $\gamma$ autoantibodies are defined as detectable anti-IFN- $\gamma$ autoantibodies in plasma diluted 1:5000 [13, 14]. Regardless, the origin of anti-IFN- $\gamma$ autoantibodies and its mechanisms remain unclear. Cases of patients with disseminated NTM because of anti-IFN- $\gamma$ autoantibodies were first reported in 2004 [15, 16]. Patel et al. recruited 23 nonimmunocompromised patients with disseminated NTM infection. Anti-IFN- $\gamma$ autoantibodies were detected in 6 of the 23 patients (26\%). All 6 patients with anti-IFN- $\gamma$ autoantibodies were female, parous, and of Asian origin [13]. Another study by Chi et al. enrolled 17 Chinese adults diagnosed with disseminated NTM infection who otherwise did not have HIV infection, autoimmune diseases, diabetes mellitus, liver cirrhosis, or congenital immunodeficiency and were not undergoing immunosuppressive or immunomodulation therapy. All 17 (100\%) patients tested positive for anti-IFN- $\gamma$ autoantibodies, 35\% had coinfection with salmonellosis, while $71 \%$ had herpes zoster infection [10, 17]. In the aforementioned studies, examination for anti-IFN- $\gamma$ autoantibodies suggested disseminated NTM infection in the patients without known immunological defect, especially in those of Asian origin. Recent studies have shown that treatment with rituximab could improve the prognosis of disseminated NTM infection with anti-IFN- $\gamma$ autoantibodies. Rituximab is a monoclonal antibody against the $\mathrm{B}$ cell protein CD20. It is recognised as a key cornerstone to treat diffuse large B-cell lymphoma. Rituximab may improve the recovery of IFN- $\gamma$ signalling by IFN- $\gamma$-induced STAT1 phosphorylation [14]. Although large randomised controlled trials to support this hypothesis are lacking, rituximab could be an option or rescue treatment for disseminated NTM infection in patients with anti- IFN- $\gamma$ autoantibody.

We presented a case of disseminated NTM infection in a previously healthy Asian male with anti- IFN- $\gamma$ autoantibody. Detection of anti-IFN- $\gamma$ autoantibodies may help to identify the risk of NTM infection, particularly in people of Asian origin. To date, there are no established differences in the treatments available for patients with or without anti-IFN- $\gamma$ autoantibodies. Due to patient's personal reasons, there was no serial data of titer of anti-IFN- $\gamma$ autoantibodies in our case during treatment and clinical followup. However, disseminated NTM infection with anti-IFN- $\gamma$ autoantibodies may indicate treatment failure and recurrent according to previous case reports. Rituximab may be considered as an optional treatment for refractory or recurrent NTM infection in patients with anti- IFN- $\gamma$ autoantibody, although evidence to support this is currently lacking.

\section{Abbreviations \\ AIDS: Acquired immune deficiency syndrome; BTS: British Thoracic Society; HIV: Human immunodeficiency virus; IFN- $\gamma$ : Anti-interferon- $\gamma$; MAC: Mycobacterium avium complex; MSMD: Mendelian susceptibility to mycobacterial diseases; NK: Natural killer cells; NTM: Nontuberculous mycobacteria; RLL: Right lower lobe; TB: Tuberculosis; TNF-a: Tumoral necrosis factor-a}

\section{Acknowledgements}

Not applicable

\section{Authors' contributions}

WCC was responsible for the clinical care of this patient. YKY was in charge of managing data, writing the manuscript. CLK and JYD performed the laboratory tests and optimized methodology. All authors read and approved the final manuscript.

\section{Funding}

Not applicable

\section{Availability of data and materials \\ The datasets used during the current study are available from the corresponding author on reasonable request.}

Ethics approval and consent to participate

Not applicable

Consent for publication

Witten informed consent was obtained from the patient for publication of this case report.

Competing interests

The authors declare that they have no competing interests. 


\section{Author details}

'Department of Chest Medicine, Taipei Medical University-Shuang Ho Hospital, Ministry of Health and Welfare, New Taipei City, Taiwan. ${ }^{2}$ Department of Chest Medicine, Taipei Veterans General Hospital, Taipei, Taiwan. ${ }^{3}$ Laboratory of Human Immunology and Infectious Disease, Graduate Institute of Clinical Medical Sciences, Chang Gung University, Taoyuan, Taiwan. ${ }^{4}$ Department of Nephrology, Chang Gung Memorial Hospital, Taoyuan, Taiwan. ${ }^{5}$ Institute of Emergency and Critical Care medicine and Faculty of Medicine, Medicine, School of Medicine, National Yang-Ming University, No. 201, Sec. 2, Shih-Pai Rd., Beitou District, Taipei 11217, Taiwan.

Received: 26 June 2019 Accepted: 16 October 2019

Published online: 29 October 2019

\section{References}

1. Ballarino GJ, Olivier KN, Claypool RJ, et al. Pulmonary nontuberculous mycobacterial infections: antibiotic treatment and associated costs. Respir Med. 2009;103:1448-55.

2. Shao $Y$, Chen $C$, Song $H$, et al. The epidemiology and geographic distribution of nontuberculous mycobacteria clinical isolates from sputum samples in the eastern region of China. PLoS Negl Trop Dis. 2015;9(3):e0003623.

3. Ramirez-Alejo N, Santos-Argumedo L. Innate defects of the IL-12/IFN-c axis in susceptibility to infections by mycobacteria and salmonella. J Interf Cytokine Res. 2014;34:307-17.

4. Chi CY, Lin CH, Ho MW, et al. Clinical manifestations, course, and outcome of patients with neutralizing anti-interferon- $\gamma$ autoantibodies and disseminated nontuberculous mycobacterial infections. Medicine (Baltimore). 2016;95:e3927. https://doi.org/10.1097/MD.0000000000003927.

5. Johnson MM, Odell JA. Nontuberculous mycobacterial pulmonary infections. J Thorac Dis. 2014:6:210-20.

6. Valour F, Perpoint T, Sénéchal A, et al. Interferon- $y$ autoantibodies as predisposing factor for nontuberculous mycobacterial infection. Emerg Infect Dis. 2016;22:1124-6.

7. Haworth CS, Banks J, Capstick T, Fisher AJ, Gorsuch T, Laurenson IF, et al. British Thoracic Society guidelines for the management of non-tuberculous mycobacterial pulmonary disease (NTM-PD). Thorax. 2017;2:ii1-ii64.

8. Fordham von Reyn C, Arbeit RD, Tosteson AN, et al. The international epidemiology of disseminated Mycobacterium avium complex infection in AIDS. International MAC Study Group. AIDS. 1996;10:1025.

9. Griffith DE, Aksamit T, Brown-Elliott BA, et al. An official ATS/IDSA statement: diagnosis, treatment, and prevention of nontuberculous mycobacteria diseases. Am J Respir Crit Care Med. 2007;175(4):367-416.

10. Chi CY, Chu CC, Liu JP, Lin CH, Ho MW, Lo WJ, et al. Anti-IFN-gamma autoantibodies in adults with disseminated nontuberculous mycobacterial infections are associated with HLA-DRB1*16:02 and HLA-DQB $1{ }^{*} 05: 02$ and the reactivation of latent varicella-zoster virus infection. Blood. 2013;121(8): 1357-66 pmid:23243276.

11. Huang S, Hendriks W, Althage A, Hemmi S, Bluethmann H, Kamijo R, et al. Immune response in mice that lack the interferon-gamma receptor. Science. 1993;259(5102):1742-5

12. Norouzi S, Aghamohammadi A, Mamishi S, Rosenzweig SD, Rezaei N. Bacillus Calmette-Guerin (BCG) complications associated with primary immunodeficiency diseases. J Inf Secur. 2012;64:543-54.

13. Patel SY, Ding L, Brown MR, et al. Anti-IFN-gamma autoantibodies in disseminated nontuberculous mycobacterial infections. J Immunol. 2005;175(7):4769-76.

14. Browne SK, Zaman R, Sampaio EP, Jutivorakool K, Rosen LB, Ding L, et al. Anti-CD20 (rituximab) therapy for anti-IFN-gamma autoantibody-associated nontuberculous mycobacterial infection. Blood. 2012;119(17):3933-9.

15. Doffinger R, Helbert MR, Barcenas-Morales G, et al. Autoantibodies to interferon-gamma in a patient with selective susceptibility to mycobacterial infection and organ-specific autoimmunity. Clin Infect Dis. 2004;38(1):e10-4.

16. Hoflich $C$, Sabat R, Rosseau $S$, et al. Naturally occurring anti-IFN-gamma autoantibody and severe infections with Mycobacterium cheloneae and Burkholderia cocovenenans. Blood. 2004;103(2):673-5.

17. Chi CY, Lin CH, Ho MW, Ding JY, Huang WC, Shih HP, et al. Clinical manifestations, course, and outcome of patients with neutralizing antiinterferon-gamma autoantibodies and disseminated nontuberculous mycobacterial infections. Medicine (Baltimore). 2016;95(25):e3927.

\section{Publisher's Note}

Springer Nature remains neutral with regard to jurisdictional claims in published maps and institutional affiliations.

\section{Ready to submit your research? Choose BMC and benefit from:}

- fast, convenient online submission

- thorough peer review by experienced researchers in your field

- rapid publication on acceptance

- support for research data, including large and complex data types

- gold Open Access which fosters wider collaboration and increased citations

- maximum visibility for your research: over $100 \mathrm{M}$ website views per year

At BMC, research is always in progress.

Learn more biomedcentral.com/submissions 